\title{
Effect of inserted sphere size on heat transfer characteristics of FCC structured pebble bed in a HTGR
}

\author{
Leisheng Chen ${ }^{1, *}$, and Jaeyoung Lee ${ }^{2}$ \\ ${ }^{1}$ ECollege of Mechanical \& Electrical Engineering, Shaanxi University of Science \& Technology, Xi' an, 710021, China \\ ${ }^{2}$ Department of Mechanical \& Control Engineering, Handong Global University, Pohang, 37554, South Korea
}

\begin{abstract}
Hot spots appearing in an operating high temperature gas-cooled reactor (HTGR) core have been considered as the most possible reason leading to a severe accident like fission production releasing to the environment, therefore, investigation on their positions and thus seeking ways to reduce the possibility of their appearance have attracted scientists' attention. In our previous studies, heat transfer characteristics of a face-centered-cubic (FCC) structured pebble-bed have been discussed, and a correlation on heat transfer coefficient with Reynolds number was presented. In this study, a method, placing a small sphere in the gap area, which is able to enhance the convective heat transfer was proposed and the effect verified as well. The influence of the sphere diameter on heat transfer performances was investigated in details. It is concluded through results analysis that (1) inserted sphere lowered the local surface temperature of adjacent pebbles by varying surrounding flow field;(2) maximum velocity of the fluid and average heat transfer coefficient increased with sphere diameter, particularly, comparing with no small sphere case, $12.95 \%$ enhancement was achieved. Such findings may provide data and information for reactor designers, and help to develop a safer HTGR pebble-bed core.
\end{abstract}

\section{Introduction}

Pebble-bed type high temperature gas-cooled reactor is considered as a candidate reactor for the very high temperature reactor (VHTR) which is one of the Generation-IV reactor types and could be used for hydrogen production because of high outlet temperature of the coolant. Its design concept exhibits excellent safety features due to the low power density and a large amount of graphite present in the core which gives large thermal inertia in an accident such as loss of coolant. However, the possible appearance of hot spots on pebble surfaces may affect the integrity of the pebbles and cause a serious accident like fission products releasing[1], which has driven researchers to investigate flow patterns of the coolant $[2,3]$ and to observe the temperature profiles of structured pebble-beds by simulations $[4,5]$. Experimentally, measurement of pebble surface temperature has been performed by Chen and Lee [6] in a face-centered-cubic (FCC) pebble-bed, in that study, heat transfer characteristics were analyzed and possible hot spots locations pointed out. Furthermore, based on the experimental results, a CFX model was created and validated as a tool to investigate the flow and thermal fields in the pebble-bed [7]. In fact, the above studies suggested the hot spots originated from pebbles contacting one another, which is obviously inevitable in a reactor core. However, if the convective heat transfer is enhanced, then temperatures of the hot spots will be lowered down, which helps to sustain the integrity of pebbles and safety of the reactor as well. Therefore, in this present study, a method of enhancing heat transfer in an FCC structured pebble-bed was proposed for the first time and its effect was examined through simulations. The results indicated that placing an additional small sphere in the gap formed by adjacent pebbles improved heat transfer performance, in addition, average heat transfer coefficient of the pebble bed increased as the additional sphere got larger. This study showed the possibility to optimize the heat transfer in a pebble-bed and might contribute to a better design of an HTGR core.

\section{Modelling and analysis}

CFX model, including turbulence model, fluid inlet temperature, heat power density, which was created previously [7] is being used in the present study. That model has been validated by experimental results, and the only difference exists in geometry part, that is a small sphere without heat generation with changeable diameter is placed in the gap formed by pebbles, therefore it is believed even with such a small change the model is still applicable to this study.

\subsection{Geometry}

Geometry is shown in Fig. 1 and parameters are listed in Table 1. The geometry consists of the fluid part and the 
solid part; the duct stands for the fluid, and the solid part is formed by 14 pebbles, 14 heaters, and 1 small sphere.

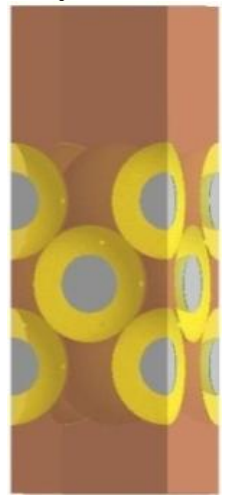

(a)

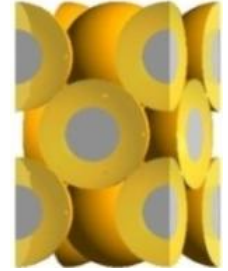

(b)
Fig. 1. Geometry of (a) FCC pebble-bed and (b) the solid part

Table 1. Parameters of geometry components

\begin{tabular}{cccccc}
\hline & Fluid & & \multicolumn{3}{c}{ Solid } \\
\hline $\begin{array}{c}\text { Width } \\
{[\mathrm{m}]}\end{array}$ & $\begin{array}{c}\text { Depth } \\
{[\mathrm{m}]}\end{array}$ & $\begin{array}{c}\text { Height } \\
{[\mathrm{m}]}\end{array}$ & Pebble & Heater & Sphere \\
\hline 0.17 & 0.17 & 0.5 & 0.12 & 0.06 & $<0.025$ \\
& & $\begin{array}{c}0.5 \\
\text { Quantity }\end{array}$ & 14 & 14 & 1 \\
\hline
\end{tabular}

As for the position of the small sphere, Fig. 2 shows more details. Only cases of $\mathrm{D}=0,0.03$, and $0.05 \mathrm{~m}$ are put here to make the concept be clearly understood, and two more cases $(\mathrm{D}=0.02,0.04 \mathrm{~m})$ were simulated during the analysis process. The rule is the way of placing a small sphere has to be based on reality, which means small sphere has to contact with at least 3 surrounding pebbles. If the sphere is too small, it is impossible to make it touch 3 pebbles, therefore, spheres with diameter less than $0.01 \mathrm{~m}$ are not considered in this study.
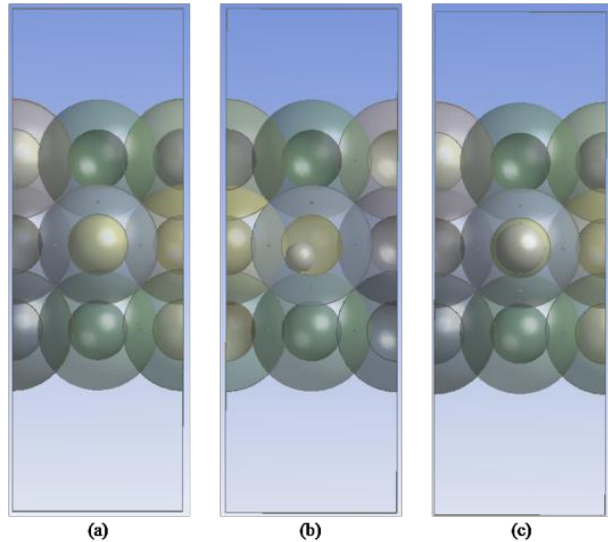

Fig. 2. Geometry with small sphere diameter of (a) $0 \mathrm{~m}$ (b) $0.03 \mathrm{~m}(\mathrm{c}) 0.05 \mathrm{~m}$

\subsection{Meshing}

It is worth noting that since thermal field one/beneath the pebble surface is strongly influenced by the flow pattern and velocity, therefore in order to observe both fields and verify their coupling mechanism, boundary layers were applied to both sides of a pebble surface, as shown in Fig. 3.

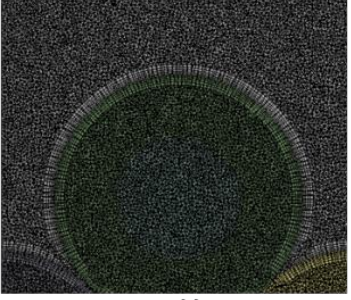

(a)

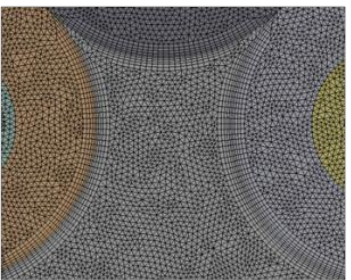

(c)

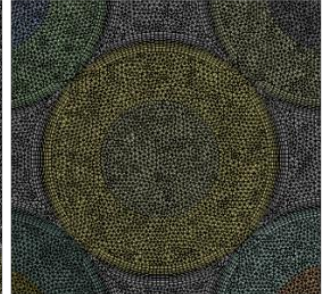

(b)

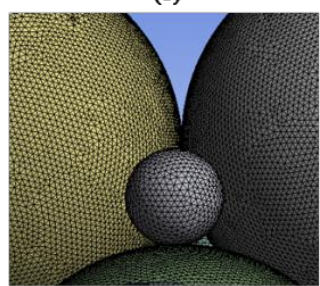

(d)
Fig. 3. Meshing of (a) the fluid part (b) pebble and heater (c) fluid-solid interface (d) small sphere

Selected element size of each component and total number of nodes and elements are put in Table 2.

Table 2. Element size and numbers.

\begin{tabular}{cccccc}
\hline \multicolumn{3}{l}{ Element size $[\mathrm{m}]$} & \multicolumn{2}{c}{ Numbers } \\
\hline Fluid & Pebble & Heater & Sphere & Nodes & Elements \\
\hline $2 \times 10^{-3}$ & $2 \times 10^{-3}$ & $2 \times 10^{-3}$ & $2 \times 10^{-3}$ & $\sim 3 \mathrm{M}$ & $\sim 14.7 \mathrm{M}$ \\
\hline
\end{tabular}

\subsection{Boundary conditions}

Boundary conditions including coolant inlet velocity, inlet temperature, and heat power density are listed in Table 3. Therein, inlet velocity and power density were calculated from original HTR-PM operation parameters according to the similarity principle.

Table 3. Boundary conditions.

\begin{tabular}{cc}
\hline Turbulence model & $\mathrm{k}-\varepsilon$ \\
Turbulence intensity & $5 \%$ \\
Boundary layers & 5 \\
Power density & $1 \times 10^{6} \mathrm{~W} / \mathrm{m}^{3}$ \\
Inlet velocity & $2.1 \mathrm{~m} / \mathrm{s}$ \\
Inlet temperature & $298.15 \mathrm{~K}$ \\
Pressure & $1 \mathrm{~atm}$ \\
\hline
\end{tabular}

\subsection{Measurement locations}

Comparing with the previous measurement positions, coordinates of No. 1, 6,7,17,18, and 23 have been shifted by $2^{\circ}$ in order to avoid pebble-pebble and pebble-sphere contact points, since heat transfer at those points is not in a way of convection but conduction. In addition, to make the results convincible, another set of 23 measurement lines as shown in Fig. 4 was applied since the pebble-bed structure was no longer symmetric when a small sphere was placed in. 


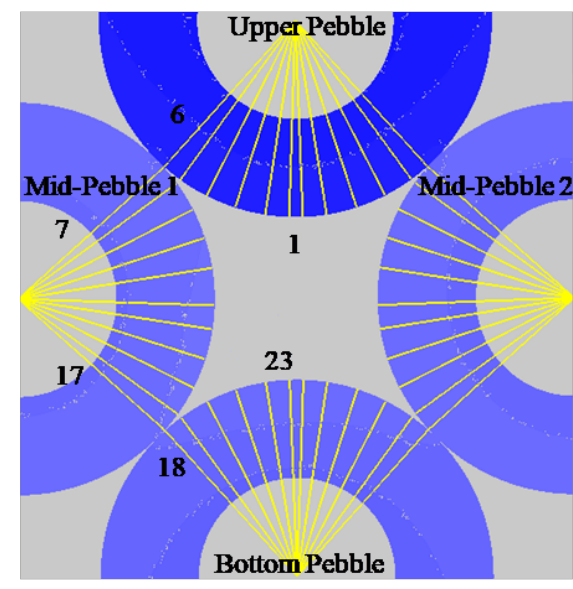

Fig. 446 temperature measurement lines

In terms of heat transfer coefficient (HTC) calculation, a second temperature is necessary besides the surface temperature, therefore temperature at $10 \mathrm{~mm}$ beneath the surface was obtained at each position. The average HTC is calculated using the following equation:

$$
h_{\text {avg }}=\sum_{i=1}^{46}\left[\frac{k_{\text {solid }} \Delta T_{i}}{r^{2}\left(\frac{1}{r_{2}}-\frac{1}{r_{1}}\right)} \times \frac{1}{T_{\text {surf }}-T_{\infty}} \times \frac{1}{46}\right]
$$

Where $\mathrm{k}$ is thermal conductivity; $\mathrm{T}$, temperature; $\mathrm{r}$, $r_{1}$, and $r_{2}$ are the radius of the pebble, distance from pebble center to surface, and distance from pebble center to $10 \mathrm{~mm}$ under the surface respectively; subscript avg and surf denote average value and surface; $i$ means the $i^{\text {th }}$ measurement point.

\section{Results and discussion}

The effect of inserted sphere size on heat transfer performances was investigated according to simulation results. Out of total 5 cases, 3 representative cases $(D=0$, $0.03,0.05 \mathrm{~m}$ ) are particularly analyzed. Velocity and thermal fields of the fluid, surface and inside temperatures and heat transfer coefficients were obtained for each case. To simplify analysis processes, one representative plane ( $\mathrm{YZ}$ plane at $\mathrm{X}=0.085 \mathrm{~m}$ ) was selected as the target of this study.

\subsection{Velocity field in the gap}

Velocity field formed in the gap area is shown in Fig. 5. Comparing cases with and without small sphere, It can be easily seen that placing a sphere in has significantly reduced the stagnant area (Blue zones in Figure 5a), which were found typically existing on top and bottom of a pebble [6]. Besides, the maximum velocity of the fluid increases and the flow field varies as the small sphere gets bigger. Down the contact points and near the side vertex are the fast flowing areas when there is no small sphere placed, but fast flowing areas are migrated after the sphere is being inserted as can be seen in Fig. $5 \mathrm{~b}, 5 \mathrm{c}$. It is worth mentioning that from the case of
$\mathrm{D}=0.03 \mathrm{~m}$, velocity field at the side where the small sphere was placed is stronger than that at the other. Therefore, such stagnant zones reduction and velocity increasing induced by small sphere will greatly improve heat transfer performances between the pebble and fluid, and then substantially reduce the possibility of hot spots appearance.

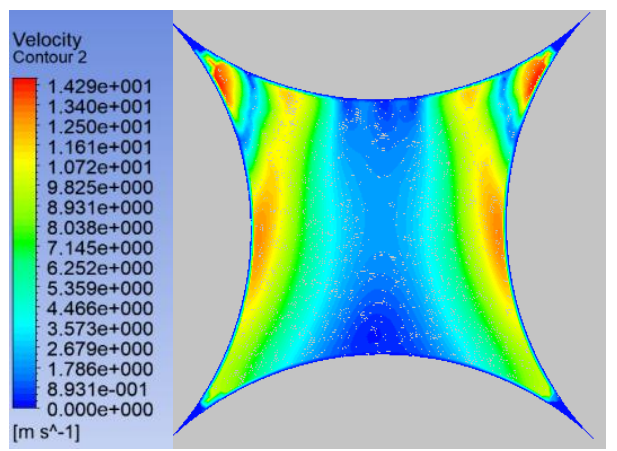

(a)

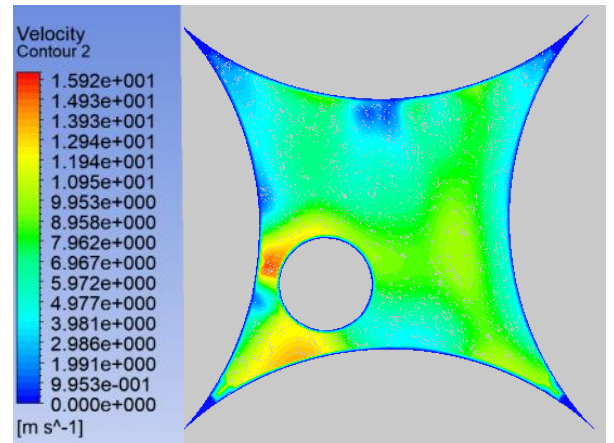

(b)

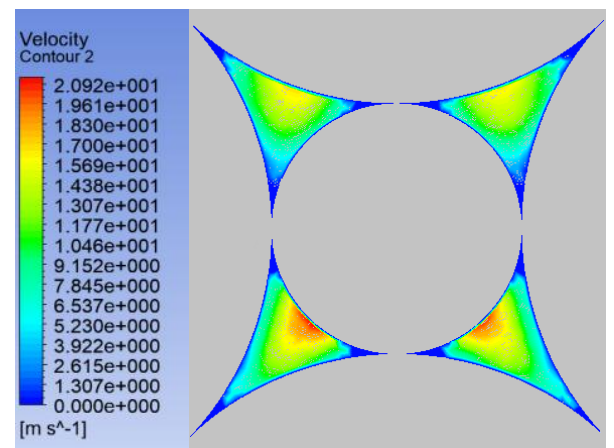

(c)

Fig. 5 Velocity field of the case with small sphere diameter at (a) $0 \mathrm{~m}$ (b) $0.03 \mathrm{~m}$ (c) $0.05 \mathrm{~m}$

\subsection{Temperature field in the gap}

Temperature of the coolant is originated from the pebble and influenced by it, as shown in Fig. 6, the upper side temperature is higher, which is because the upper pebble has the relatively higher temperature than the middle and bottom pebbles. The maximum temperature is found near the upper pebble, as the fluid flows, it gets cooled down. It is really difficult to tell how overall temperature profile is affected by the small sphere only based on these 3 figures at this moment, thus, pebble surface temperatures at positions shown in Fig. 4 were obtained and their variation trends were analyzed in details. 


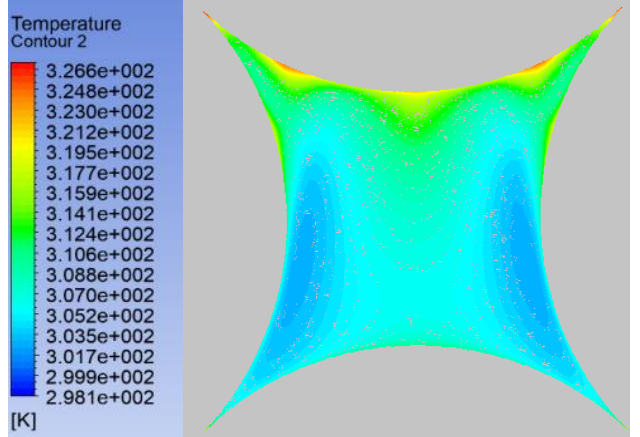

(a)

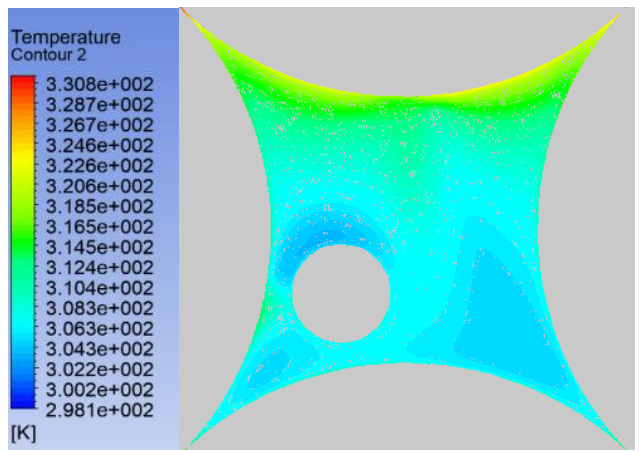

(b)

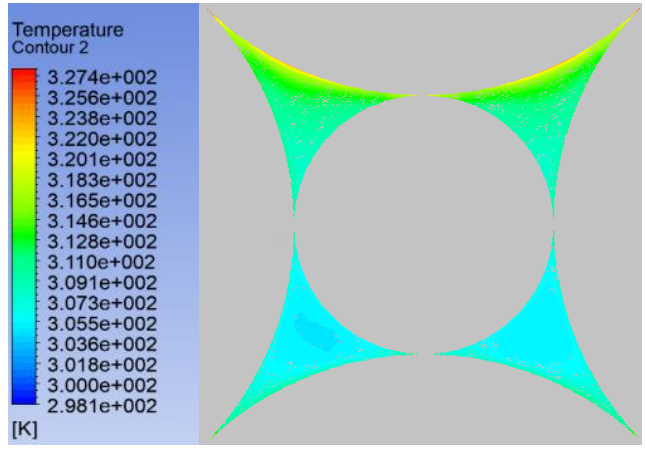

(c)

Fig. 6 Temperature field of the case with small sphere diameter at (a) $0 \mathrm{~m}$ (b) $0.03 \mathrm{~m}$ (c) $0.05 \mathrm{~m}$

\subsection{Surface temperature of pebbles}

Surface temperatures of the pebbles are shown in Fig. 7. The upper pebble is not likely to be strongly influenced by the small sphere, and only the vertex shows a slight decrease when the sphere is with a diameter of $0.05 \mathrm{~m}$. The middle and bottom pebbles show fierce variations comparing cases with and without small sphere. Temperature decreases as the sphere grows bigger, particularly, such a trend is found with the bottom pebble. The variation trend indicates that inserted small sphere lowers surface temperatures of adjacent pebbles, which suggests inserting small sphere reduce the possibility of hot spots appearance, however, it is still not sure that whether it is by improving convective heat transfer or not. For cases of $\mathrm{D}=0$ and $\mathrm{D}=0.05 \mathrm{~m}$, the pebble bed is symmetric, that is why temperatures of half measurement positions are in good agreement with that of the other half. In terms of case $\mathrm{D}=0.03 \mathrm{~m}$, due to effect of the small sphere, temperatures of the half with sphere are lower than those of the other half.

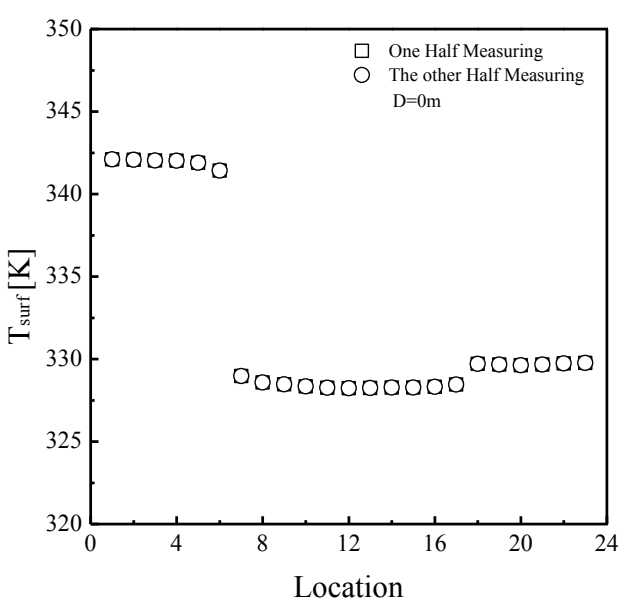

(a)

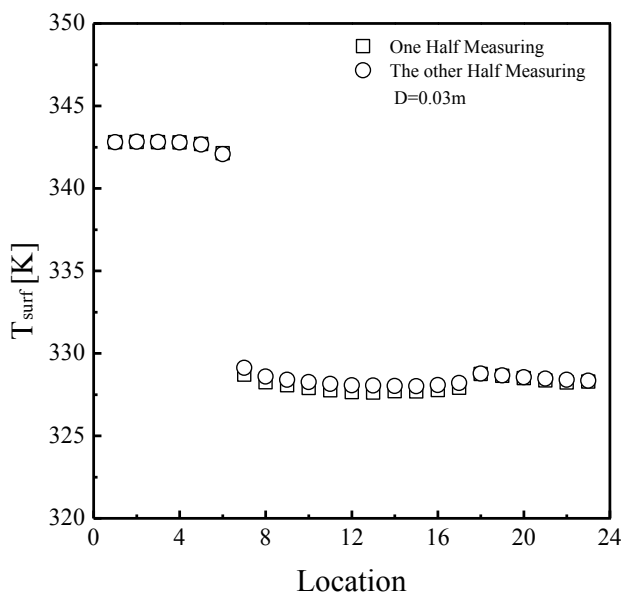

(b)

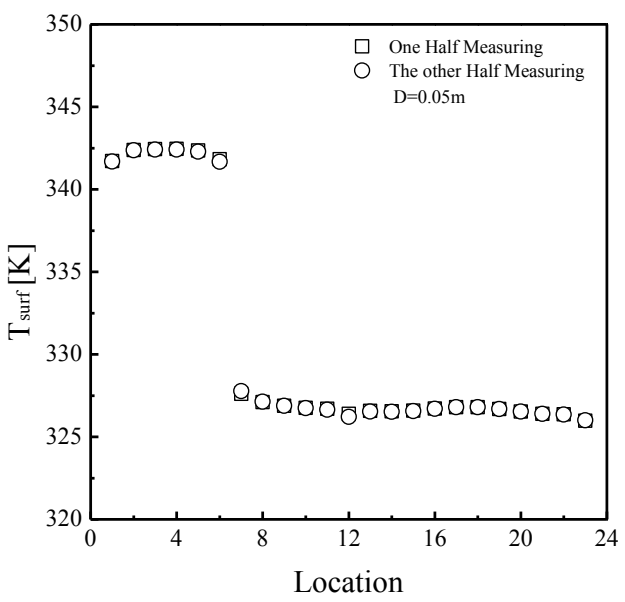

(c)

Fig. 7 Surface temperature of pebbles under 3 cases of (a) $D=0 m$ (b) $\mathrm{D}=0.03 \mathrm{~m}$ (c) $\mathrm{D}=0.05 \mathrm{~m}$ 


\subsection{Heat transfer coefficient analysis}

Following the temperature measurement at 46 positions, local heat transfer coefficient at the same positions was obtained as well in order to evaluate the effect of small sphere on heat transfer characteristics. 2 extra cases $(\mathrm{D}=0.02,0.04 \mathrm{~m})$ together with the previous 3 cases were considered, and the results are shown in Fig. 8.

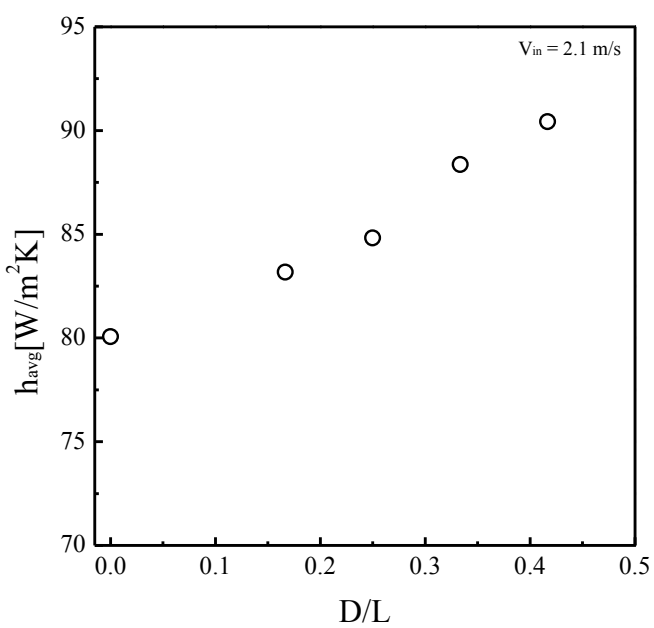

Fig. 8 Average heat transfer coefficient of all cases under coolant inlet velocity of $2.1 \mathrm{~m} / \mathrm{s}$

It can be easily seen that average heat transfer coefficient increases with the sphere diameter ( $\mathrm{L}$ is the characteristic length, $0.12 \mathrm{~m}$ ), comparing with the case without sphere, heat transfer was enhanced by $12.95 \%$ if a sphere with a diameter of $0.05 \mathrm{~m}$ was placed in. Since $0.05 \mathrm{~m}$ is the biggest diameter a sphere can have in an FCC structured pebble-bed, $12.95 \%$ will be the largest enhancement that can be obtained under conditions considered in this study. One thing that needs to be taken into account is the newly added sphere has a different size from fuel elements, and the stress imposed on it may be bigger than that imposed on fuel pebbles. Therefore, in order to apply such method into a real HTGR pebblebed, stress analysis on the sphere is required and certainly necessary. Acquiring a diameter with it a sphere can help pebble-bed have the best heat transfer performance, meanwhile, be able to sustain its integrity under extremely heavy load condition, which is the nextstep work.

\section{Conclusions}

Effect of an inserted small sphere on the heat transfer characteristics of an FCC structured pebble-bed has been investigated, therein, sphere diameter was considered as a parameter that influenced heat transfer performance. Velocity and thermal fields in the gap were obtained, in addition, the surface temperatures of certain pebbles were measured. Furthermore, the average heat transfer coefficient of the pebble-bed with small sphere under inlet velocity of $2.1 \mathrm{~m} / \mathrm{s}$ was acquired. It was found that:
(1) the maximum velocity of fluid increased with sphere diameter; (2) surface temperatures of the middle and bottom pebbles lowered down as sphere got bigger, particularly, temperatures of the half side with a sphere were lower than those of the other half; (3) average heat transfer coefficient increased as sphere diameter varied from 0 to $0.05 \mathrm{~m}$, and the largest enhancement comparing with case of no sphere placed was obtained as $12.95 \%$. It is believed that these findings have provided one more solution sustaining safe HTGR operation and will help design a better pebble-bed.

This work was supported by start-up young researchers fund (2019BJ-07), granted by Shaanxi University of Science\&Technology.

\section{References}

1. R.Moormann, Jul-4275, Julich, (2008)

2. Y.A.Hassan, Nucl. Eng. Des. 238, 530-537 (2008)

3. L.S.Chen, W.R.Lee, J.Y.Lee, Proceedings of PBNC2016, 3, 399-409 (2016)

4. A.Shams, F.Roelofs, E.M.J.Komen, E.Baglietto, Nucl. Eng. Des., 263, 490-499 (2013)

5. Y.M.Ferng, K.Y.Lin, Nucl. Eng. Des., 258, 66-75 (2013)

6. L.S.Chen, J.Y.Lee, Anna. Nucl. Energy 110, 338-348 (2017)

7. L.S.Chen, W.R.Lee, J.Y.Lee, Appl. Therm. Eng. 121, 473-483 (2017) 